\title{
Fasting and Non-Fasting Lipid Profile among Health Care Workers at Teaching Hospital Batticaloa SriLanka
}

\author{
Maheswaran Umakanth ${ }^{* *}$, Majitha Ibrahim² \\ ${ }^{1}$ Faculty of Health Care Sciences, Eastern University, Vantharumoolai, Eastern Province, Sri Lanka \\ ${ }^{2}$ Teaching Hospital, Batticaloa, SriLanka \\ Email: "mumakanth1972@gmail.com
}

How to cite this paper: Umakanth, M. and Ibrahim, M. (2018) Fasting and Non-Fasting Lipid Profile among Health Care Workers at Teaching Hospital Batticaloa SriLanka. Journal of Biosciences and Medicines, 6, 15-22.

https://doi.org/10.4236/jbm.2018.67002

Received: June 19, 2018

Accepted: July 14, 2018

Published: July 17, 2018

Copyright $\odot 2018$ by authors and Scientific Research Publishing Inc. This work is licensed under the Creative Commons Attribution International License (CC BY 4.0).

http://creativecommons.org/licenses/by/4.0/

\section{(c) (i) Open Access}

\begin{abstract}
Requesting patients to come with long fasting state (12 - 14 hours) for lipid profile measurements are a major inconvenience. However, most blood tests, even glycemic management, can be done in a non-fasting state, for example by requesting an $\mathrm{HbA1C}$. It is understandable that when we order lipid profile test, laboratories are very rigid on fasting $(12-14 \mathrm{~h})$ or refuse to do the test if fasting is not enough. To answer these delusions, we conducted a cross-sectional study among the health care workers at Teaching Hospital Batticaloa, SriLanka, after an overnight fast (12 - 14 hours) blood taken for lipid profile. Following weeks, we collected blood from the same healthcare workers, after breakfast $(2-4$ hours after meal). In this study, measurements of fasting lipid profile, including high-density lipoproteins (HDL), low-density lipoproteins (LDL), total cholesterol (TC), triglyceride (TG) and non-HDL significantly $(p<0.05)$ differ from non-fasting lipid profile measurement. The mean difference in lipid profile in fasting versus non-fasting among healthcare workers was $34.13 \mathrm{mg} / \mathrm{dl}$ for TG, $-5.65 \mathrm{mg} / \mathrm{dl}$ for total TC, $-1.94 \mathrm{mg} / \mathrm{dl}$ for HDL-cholesterol, $3.71 \mathrm{mg} / \mathrm{dl}$ for non-HDL and $12.3 \mathrm{mg} / \mathrm{dl}$ for LDL-cholesterol. This momentous change of different meanings does not play any significant role in cardiovascular risk assessment. However, a patient with a family history of the premature atherosclerotic cardiovascular disease (ASCVD), or familial hyperlipidemia, screening and follow-up should preferably be performed with fasting.
\end{abstract}

\section{Keywords}

Non-Fasting Lipid Profile, Fasting Lipid Profile 


\section{Introduction}

Lipids are a group of fats and fat-like substances that are a vital ingredient of our cells. It is also main sources of energy. Measuring lipid profile is a routine test to evaluate the cardiovascular risk, given the high correlation of hypercholesterolemia and cardiovascular risk. There are two main types of lipids, total cholesterol (TC) and triglyceride (TG) which are combined with transport protein called lipoprotein. These particles measured with lipid profile and classified based on these densities called, low-density lipoproteins (LDL), high-density lipoproteins (HDL) and very low-density lipoproteins (VLDL). However, in most of the laboratory, LDL-cholesterol is calculated from the Friedewald equation (LDL $=$ Total cholesterol (TC)-HDL-TG/5). This finding is well connected with a standard test of LDL by ultracentrifugation when TG is less than $400 \mathrm{mg} / \mathrm{dl}$ [1].

This Friedewald equation method is unacceptable if TG values are above 400 $\mathrm{mg} / \mathrm{dL}$. In such circumstances, direct quantity of LDL is indicated. In addition, this is only applicable for calculating LDL cholesterol in an overnight fasting specimen. In real clinical scenario calculated LDL cholesterol may not reflect true LDL cholesterol levels. If there is a discrepancy between measured LDL cholesterol level and calculated LDL cholesterol level, it indicates that there is a modification of lipoprotein metabolism [1].

However, measuring lipid profile in the non-fasting state becomes popular among clinical practice, in part because 12 - 14 hours fasting is uncomfortable especially, the elderly and patients who had diabetes. Moreover, a prandial state lipid profile is more honest for calculating total atherogenic lipoproteins because it includes the hepatic origin in the fasting state and intestinal origin in the non-fasting state. Supplementary, data has aroused in recent years that most conspicuously from Scandinavian countries, there is not much difference between fasting and non-fasting lipid values. As a result, increasing number of professional societies and organizations now recommend using non-fasting lipid profile levels to establish the cardiovascular risk state [2]. The aim of this study is to critically evaluate the clinical implications of the use of non-fasting rather than fasting lipid profiles.

\section{Methods}

This is a cross-sectional study conducted among health care workers, in which random non-fasting lipid profile have been compared with their fasting condition, at Teaching Hospital Batticaloa, SriLanka. Approval was taken from the director, Teaching Hospital Batticaloa to conduct this study. As a part of the non-communicable screening programme, almost all health workers were screened for risk assessment, including fasting blood sugar and lipid profile test. Healthcare workers were explained the advantages and disadvantages of non-fasting lipid profile test. Those who were voluntarily participated for both fasting and non-fasting lipid tests were included in this study. Health workers with age of 25 to 60 -year were included in this study. The written consent was taken from all the participants. After an overnight (12 - 14 hours) fast, blood 
samples were taken for lipid profile. Within a week, we collected non-fasting blood samples ( 2 - 4 hours) after breakfast. Time since last meal was gained by self-administered questionnaire from the patients at the time of testing. Fasting with less than 12 hours or more than 14 hours was excluded from this study. Similarly, non-fasting with less than 2hours or more than 4 hours were excluded. All blood samples were analyzed at Teaching hospital Batticaloa, SriLanka where, using standard laboratory protocol. High-density lipoprotein cholesterol, total cholesterol and triglyceride levels were measured directly; LDL cholesterol levels were estimated using the Friedewald equation.

The data were analyzed for normality using Anderson-darling, Kolmogorov-Smirnov and Shapiro-Wilk tests. The TC, HDL and LDL followed the normal distribution for all normality test $(p>0.05)$, while TG level did not follow the normal distribution for all normality test $(p<0.05)$. Therefore, the parametric analysis (paired t-test) was performed to total cholesterol (TC), HDL and LDL and non-parametric analysis (Wilcoxon Signed Ranks Test) was performed to triglyceride (TG) to compare them before and after a meal. All the analysis was done in $95 \%$ of confidence interval. The outliers of the parameters were removed before the significant test. All the analysis was performed using R statistical software.

\section{Results}

Total 84 health works participated in both fasting and non-fasting lipid profile test. Of these, $54(64.28 \%)$ were male and $30(35.71 \%)$ were female. The mean fasting TC, HDL, LDL, non-HDL cholesterol measurements are higher than non-fasting measurements, the fasting measurements are $194.99 \mathrm{mg} / \mathrm{dl}$ for TC, $46.05 \mathrm{mg} / \mathrm{dl}$ for HDL, $123.43 \mathrm{mg} / \mathrm{dl}$ for LDL, and $148.94 \mathrm{mg} / \mathrm{dl}$ for non-HDL cholesterol (Figure 1). The mean non-fasting measurements are $189.34 \mathrm{mg} / \mathrm{dl}$ for TC, $44.11 \mathrm{mg} / \mathrm{dl}$ for HDL, $111.13 \mathrm{mg} / \mathrm{dl}$ for LDL, and $145.23 \mathrm{mg} / \mathrm{dl}$ for non-HDL cholesterol. However, mean non-fasting TG is higher than fasting measurements, $176.53 \mathrm{mg} / \mathrm{dl}$, and $132.40 \mathrm{mg} / \mathrm{dl}$ respectively (Table 1 ) (Figure 1).

Random non-fasting lipid profile have been compared with their fasting condition in which the TC, HDL-cholesterol, and non-HDL cholesterol showed the significant $(P<0.05)$ difference with the mean difference of $-5.65 \mathrm{mg} / \mathrm{dl},-1.94$ $\mathrm{mg} / \mathrm{dl}$, and $-3.71 \mathrm{mg} / \mathrm{dl}$, respectively but with moderate effect (Figure 1).

Table 1. Non-fasting and fasting lipoprotein value and significant test of different lipoprotein components.

\begin{tabular}{cccccc}
\hline Cholesterol & Fasting(mg/dl) & Non-fasting(mg/dl) & T value & $p$-Value & Effect size $^{*}$ \\
\hline TC & 194.99 & 189.34 & $\mathrm{t}(70)=4.3295$ & $p<0.05$ & Moderate (0.58) \\
HDL & 46.05 & 44.11 & $\mathrm{t}(81)=5.2829$ & $p<0.05$ & Moderate (0.58) \\
LDL & 123.43 & 111.13 & $\mathrm{t}(76)=8.734$ & $p<0.05$ & Large $(0.99)$ \\
Non-HDL & 148.94 & 145.23 & $\mathrm{t}(82)=3.405$ & $p<0.05$ & Moderate $(0.58)$
\end{tabular}

${ }^{\star}$ Effect size of difference was calculated using Cohen's d with Hedges correction. 


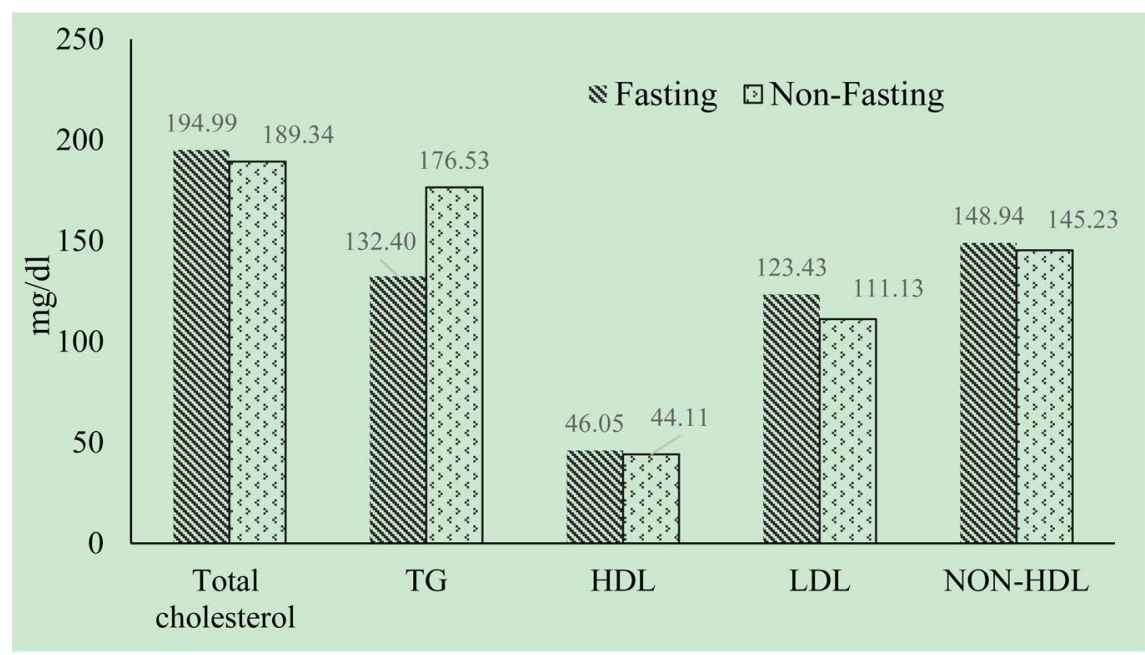

Figure 1. Compare the fasting and non-fasting mean value of Total cholesterol, Triglyceride (TG), high density lipoprotein (HDL), low density lipoprotein (LDL) and non-HDL.

However, the LDL also showed significant $(P<0.05)$ difference but with large effect size, and with the mean difference of $-12.3 \mathrm{mg} / \mathrm{dl}$ (Table 1). Furthermore, Wilcoxon Signed Ranks Test showed that there was a significant difference in triglyceride measurements as well $(\mathrm{v}=191.5, p<0.001$, Hedges correction $=$ -0.89 , which is largest effect size). The mean difference of TG, between random fasting and fasting was $+34.13 \mathrm{mg} / \mathrm{dl}$.

\section{Discussion}

Remarkably, the evidence is deficient that fasting is superior to non-fasting lipid profile tests. Fasting lipid profile is ideal to reduce the variability at the time of sample collection [3]. Quite a lot of studies suggest that the measurement of lipid profile test in non-fasting state is an acceptable substitute [4]. However, measurement of a random non-fasting lipid profile also able to divulge individual metabolic abnormalities in lipid clearance. Furthermore, there are advantages to using random non-fasting samples rather than fasting samples for measuring the lipid profile [5]. It is interesting that since 2009, non-fasting lipid profile test has been practicing as a standard blood test in Denmark. However, clinicians from Denmark agreed to re-check fasting triglyceride if non-fasting triglyceride measurement is more than $350 \mathrm{mg} / \mathrm{dl}$ (4 mmol/l) [6].

The foremost worry of physicians regarding random lipid profile test is the variable effect after a meal. It is obvious that several studies cited that increasing level of TG concentration has been seen after a meal [7]. A Similar pattern has been observed in our study as well. In fasting mean TC, HDL, LDL, Non-HDL cholesterol measurements are $194.99 \mathrm{mg} / \mathrm{dl}, 46.05 \mathrm{mg} / \mathrm{dl}, 123.43 \mathrm{mg} / \mathrm{dl}$, and $148.94 \mathrm{mg} / \mathrm{dl}$ respectively (Figure 1). However, mean random non-fasting mean value of TC, HDL, LDL, non-HDL cholesterol measurements are slightly lower than fasting measurements. As this lipids fraction are normally distributed, we performed paired T-test which revealed that significant $(P<0.05)$ different has 
been observed in the measurements of TC, HDL, LDL, and non-HDL cholesterol. Furthermore, in 2017, a similar study reported that mean difference in lipid profile measurements in non-fasting versus fasting patients are $26 \mathrm{mg} / \mathrm{dL}$ for TG, $-8 \mathrm{mg} / \mathrm{dL}$ for TC, $-8 \mathrm{mg} / \mathrm{dL}$ for LDL cholesterol, $+8 \mathrm{mg} / \mathrm{dL}$ for remnant cholesterol, and $-8 \mathrm{mg} / \mathrm{dL}$ for non-HDL cholesterol [8]. According to this study, non-fasting lipid profile has an equal prognostic ability for cardiovascular disease and should not affect treatment decision. In our study, even though significant difference observed both non-fasting and fasting lipid profile measurements, however mean difference in both non-fasting and fasting lipid profile are more or less similar to a study conducted by Nordestgaard, $B G$ [8]. In our study, $34.13 \mathrm{mg} / \mathrm{dl}$ for triglyceride, $-5.65 \mathrm{mg} / \mathrm{dl}$ for TC, $-1.94 \mathrm{mg} / \mathrm{dl}$ for HDL-cholesterol, $-3.71 \mathrm{mg} / \mathrm{dl}$ for non-HDL and $-12.3 \mathrm{mg} / \mathrm{dl}$ for LDL-cholesterol. This mean different not causing any clinical significant, when we assess cardio vascular risk state.

However, triglyceride level (TG) more significantly $(P<0.05$ and Hedges correction $=-0.89$ ) higher in random non-fasting than fasting state. A study conducted in China which revealed that postprandial TG level higher than fasting sample, however peak increment has been observed 0.5 hours after lunch and also stated that changes in TC, HDL, LDL and apo B were not significantly different from fasting lipid measurement [9]. A similar study stated that the variances in random non-fasting lipid changes were related to individual enzyme and hormone levels [2] [10]. However, most of the studies have shown that lipid profile levels minimally change with a meal including, tiny changes for HDL cholesterol, minor changes for TC, and non-HDL and modest changes for TG [11].

Though, cardiovascular risk assessment and monitoring of lipid-lowering based on LDL-cholesterol and TG should be performed in a blood sample collected after a period of 12 - 14 hours of overnight fasting without any caloric intake except for water and medications. The reason behind that is non-fasting lipid profile measurement show elevated TG, which in turn change the ratio of TG to total cholesterol and invalidate the Friedewald method, leads to overestimating the LDL-cholesterol. In this situation, a direct assay for LDL-cholesterol can be used. Moreover, weight has been placed on non-HDL-cholesterol, (TC-HDL) as a secondary target of therapy [12]. Introduce the lipid-lowering therapy mainly depends on the number of coronary heart disease (CHD) risk factors and baseline LDL and or Non-HDL cholesterol [13]. Though, non-fasting level of TG measurement is higher than fasting. In our study mean difference of TG was $34.13 \mathrm{mg} / \mathrm{dl}$. However, the clinical point of view treatment should be started if TG more than $500 \mathrm{mg} / \mathrm{dl}$. Random non-fasting elevation of TG, does not affect the treatment goal, however if non-fasting TG is more than $450 \mathrm{mg} / \mathrm{dl}$, should be consider fasting test [9].

Most of the recently published studies emphasize that non-fasting blood sample is simple, safer, increasing efficiency and saves money. Also, number of stu- 
died cited that, non-fasting lipid profile components are better at forecasting the risk of cardiovascular events [14]. Measurement of non-fasting lipid profile clearly reveals individual metabolic abnormalities in lipid clearance, elevated non-fasting TG associated with high risk of myocardial infarction and all cause of mortality [15]. It is facts that we should be careful about the certain type of patients, especially, diabetes where fasting may mask the abnormalities in TG metabolism [16]. Recently the joint consensus statement from the European Atherosclerosis Society and European Federation of Clinical Chemistry and Laboratory Medicine suggest that non-fasting lipids become the new standard for lipid measurement [10].

Non-fasting and fasting lipid measurements should be complementary but not mutually exclusive. Furthermore, the panel transcribed that non-fasting testing is generally appropriate in patients undergoing an initial lipid profile or cardiovascular risk assessment, those who are admitted with acute coronary syndrome, and for children, the elderly, and patients with diabetes. It is also appropriate if the patient prefers it. However, in certain conditions in which a fasting lipid profile may be favored such as non-fasting TG more than $(0.5 \mathrm{mmol} / \mathrm{l}) 450$ $\mathrm{mg} / \mathrm{dl}$, known hypertriglyceridemia, recovering from hypertriglyceridemic pancreatitis, starting medications that cause severe hypertriglyceridemia, screening and follow-up should ideally be performed with fasting or combined with apolipoprotein B (apo-B) [17] [18] [19].

\section{Conclusion}

In this study, significant differences were seen between non-fasting and fasting blood samples. However, more significant difference was observed in Triglyceride. There are several studies revealed that most of the lipid profile measurements are minimally changed with meal. When we consider a range of risk categories of lipid profile, this minimal change of fractions does not alter the management plan. However, non-fasting lipids are sufficient for general screening of cardiovascular risk. However, in certain conditions in which a fasting lipid profile may be favored such as non-fasting TG more than $(0.5 \mathrm{mmol} / \mathrm{l}) 450 \mathrm{mg} / \mathrm{dl}$, known hypertriglyceridemia, recovering from hypertriglyceridemic pancreatitis, starting medications that cause severe hypertriglyceridemia, screening and follow-up should ideally be performed with fasting or combined with apolipoprotein B (apo-B). In future, further prospective studies are required to compare the association of fasting and non-fasting lipid profile from the same individuals with subsequent cardiovascular outcomes.

\section{Limitation of the Study}

There are some limitations in this study, first non-fasting only restricted to 2 - 4 hours from the breakfast. The main meal of SriLanka people is lunch, but this study only compared the fasting lipid profile with random after breakfast. Only 84 heath workers, participated both fasting and non-fasting lipid profile test, this 
is fairly small sample to come to the decision. Furthermore we didn't compare the sex and ethnic group difference. Finally, familial hypercholesterolemia subjects are not excluded from this study.

\section{Consent to Participate}

Written consent was taken.

\section{Consent for Publication}

Consent was obtained from the health care workers, Teaching Hospital Batticaloa for publication of this article.

\section{Availability of Data and Material}

All data gathered during this study are included in this published article.

\section{Conflict of Interest}

None declared.

\section{Funding}

This research received no funding support.

\section{References}

[1] Nordestgaard, B.G., Langsted, A., Mora, S., et al. (2016) Fasting Is Not Routinely Required for Determination of a Lipid Profile: Clinical and Laboratory Implications Including Flagging at Desirable Concentration Cut-Points-A Joint Consensus Statement from the European Atherosclerosis Society and European Federation of Clinical Chemistry and Laboratory Medicine. European Heart Journal, 37, 1944-1958. https://doi.org/10.1093/eurheartj/ehw152

[2] Langsted, A., Freiberg, J.J. and Nordestgaard, B.G. (2008) Fasting and Nonfasting Lipid Levels Influence of Normal Food Intake on Lipids, Lipoproteins, Apolipoproteins, and Cardiovascular Risk Prediction. Circulation, 118, 2047-2056. https://doi.org/10.1161/CIRCULATIONAHA.108.804146

[3] Langsted, A., Freiberg, J.J. and Nordestgaard, B. (2008) Fasting and Non-Fasting Lipid Levels: Influence of Normal Food Intake on Lipids, Lipoproteins, Apolipoproteins, and Cardiovascular Risk Prediction. Circulation, 118, 2047-2056. https://doi.org/10.1161/CIRCULATIONAHA.108.804146

[4] Langsted, A. and Nordestgaard, B.G. (2015) Nonfasting Lipid Profiles: The Way of the Future. Clinical Chemistry, 61, 1123-1125. https://doi.org/10.1373/clinchem.2015.243139

[5] Mora, S., Rifai, N. and Buring, J.E. and Ridker, P. (2008) Fasting Compared with Nonfasting Lipids and Apolipoproteins for Predicting Incident Cardiovascular Events. Circulation, 118, 993-1001. https://doi.org/10.1161/CIRCULATIONAHA.108.777334

[6] Langsted, A. and Nordestgaard, B. (2011) Nonfasting Lipids, Lipoproteins, and Apolipoproteins in Individuals with and without Diabetes: 58,434 Individuals from the Copenhagen General Population Study. Clinical Chemistry, 57, 482-489. https://doi.org/10.1373/clinchem.2010.157164 
[7] Nayor, M. and Vasan, R.S. (2016) Recent Update to the Us Cholesterol Treatment Guidelines: A Comparison with International Guidelines. Circulation, 133, 1795-1806. https://doi.org/10.1161/CIRCULATIONAHA.116.021407

[8] Nordestgaard, B. (2017) A Test in Context: Lipid Profile, Fasting Versus Nonfasting. Journal of the American College of Cardiology, 70, 1637.

https://doi.org/10.1016/j.jacc.2017.08.006

[9] Yang, D., Cai, Q., Qi, X. and Zhou, Y. (2018) Postprandial Lipid Concentrations and Daytime Biological Variation of Lipids in a Healthy Chinese Population. Annals of Laboratory Medicine, 38, 431-439. https://doi.org/10.3343/alm.2018.38.5.431

[10] Marcovina, S.M., Gaur, V.P. and Albers, J. (1994) Biological Variability of Cholesterol, Triglyceride, Low- and High-Density Lipoprotein Cholesterol, Lipoprotein (a), and Apolipoproteins A-I and B. Clinical Chemistry, 40, 574-578.

[11] Nigam, P.K. (2011) Serum Lipid Profile: Fasting or Non-Fasting? Indian Journal of Clinical Biochemistry, 26, 96-97. https://doi.org/10.1007/s12291-010-0095-x

[12] Jellinger, P.S., Handelsman, Y., Rosenblit, P.D., Bloomgarden, Z.T., Fonseca, V.A., Garber, A.J., et al. (2017) American Association of Clinical Endocrinologists and American College of Endocrinology Guidelines for Management of Dyslipidemia and Prevention of Cardiovascular Disease. Endocrine Practice, 23, 1-87.

http://journals.aace.com/doi/10.4158/EP171764.APPGL https://doi.org/10.4158/EP171764.APPGL

[13] National Institute of Health (2001) NCEP Cholesterol Guidelines. [NCEP] National Cholesterol Education Program, 329, 925-929.

[14] Bansal, S., Buring, J.E., Rifai, N., Mora, S. and Sacks, et al. (2007) Fasting Compared with Nonfasting Triglycerides and Risk of Cardiovascular Events in Women. JAMA, 298, 309-316. https://doi.org/10.1001/jama.298.3.309

[15] Nordestgaard, B.G., Benn, M. and Schnohr, P. (2007) Nonfasting Triglycerides and Risk of Myocardial Infarction, Ischemic Heart Disease, and Death in Men and Women. JAMA, 298, 299-308. https://doi.org/10.1001/jama.298.3.299

[16] Farukhi, Z. and Mora, S. (2016) Re-Assessing the Role of Non-Fasting Lipids; A Change in Perspective. Annals of Translational Medicine, 4, 431-431. http://atm.amegroups.com/article/view/12394/12864 https://doi.org/10.21037/atm.2016.11.15

[17] Driver, S.L., Martin, S.S., Gluckman, T.J., Clary, J.M., Blumenthal, R.S. and Stone, N.J. (2016) Fasting or Nonfasting Lipid Measurements It Depends on the Question. Journal of the American College of Cardiology, 67, 1227-1234. https://doi.org/10.1016/j.jacc.2015.12.047

[18] Sniderman, A., Tremblay, A., Bergeron, J., et al. (2007) Diagnosis of Type III Hyperlipoproteinemia from Plasma Total Cholesterol, triglYceride, and Apolipoprotein B. Journal of Clinical Lipidology, 1, 256-263. https://doi.org/10.1016/j.jacl.2007.07.006

[19] Blom, D.J. and O’Neill, F.H.M.A. (2005) Screening for Dysbetalipoproteinemia by Plasma Cholesterol and Apolipoprotein B Concentrations. Clinical Chemistry, 51, 904-907. https://doi.org/10.1373/clinchem.2004.047001 